\title{
Influence de certains corps gras sur le Sun Protection Factor (SPF) mesuré in vitro
}

\author{
Céline COUTEAU \\ Stéphanie BENJAMIN \\ Céline FOUREL \\ Eva PAPARIS \\ Laurence COIFFARD \\ Laboratoire de pharmacie industrielle et \\ cosmétologie - EA 2160 (SMAB), \\ 1 rue Gaston Veil 44035 Nantes Cedex 01 \\ <laurence.coiffard@univ-nantes.fr>
}

\section{Introduction}

Suite aux expositions solaires non protégées des années 60-70, nous assistons à une progression du nombre de cancers cutanés. Actuellement, la prévention des cancers liés au soleil est importante : réglementation particulière pour les instituts dispensateurs d'UVA avec une formation obligatoire pour les esthéticiennes détentrices de solarium, sensibilisation de la population relayée par les médias (service météo...), aux risques liés à toute exposition, conseil par le pharmacien d'officine...

Pour notre part, nous nous intéressons tout particulièrement au développement de nouveaux produits solaires et étudions l'influence de l'excipient sur l'efficacité de la protection solaire. Pour cela, un certain nombre de produits cosmétiques ont été réalisés et leur SPF (Sun Protection Factor) a été déterminé in vitro. En effet, la méthode Colipa, méthode in vivo [1] qui constitue actuellement la méthode de référence pour la détermination des indices apposés sur les emballages des produits solaires, présente à notre avis 3 inconvénients majeurs : - la dose appliquée sur le sujet de $2 \mathrm{mg} / \mathrm{cm}^{2}$ est nettement supérieure à celle utilisée dans la réalité $\left(0,5\right.$ à $\left.1,5 \mathrm{mg} / \mathrm{cm}^{2}\right)$ [2];

- les doses d'UV reçues par les volontaires, au cours du test, sont loin d'être négligeables, en particulier pour les produits à forts indices ;

- les résultats varient fortement selon les individus recrutés et la saison à laquelle le test est effectué.

Dans ce travail, nous avons étudié l'influence de la nature de la phase grasse sur le SPF et nous avons ainsi testé 18 corps gras. Seule

\begin{abstract}
In order to improve the knowledge on the influence of vehicle on the SPF (Sun Protection Factor), we determined in vitro the SPF from an oil-in-water (O/W) emulsion containing octylmethoxycinnamate (4\%). We changed the oily phase composition. The in vitro determination of SPF is based on the physical determination of the reduction of the energy in the UV range, through a film of product which has previously been spread on an adequate substrate. About $30 \mathrm{mg}$ were applied on roughened PMMA plates and the transmission measurements are carried out using a spectrophotometer equipped with integrating sphere. Eighteen oily ingredients were studied. Each one of them was incorporated in the amount of $20 \%$. It is the lanolin which appears most effective SPF booster. Then oils come blackcurrant, argan, sweet almond, sesame, groundnut, carthame and palmotene.
\end{abstract}

Key words: SPF in vitro, oily ingredients, emulsion O/W

I'huile de tournesol avait été étudiée à ce jour et un certain pouvoir protecteur vis-à-vis des UVA courts a été démontré [3].

\section{Matériels et méthodes}

Le produit qui nous sert de référence est le standard FDA (homosalate $8 \%-$ SPF $=4$ ). Les crèmes étudiées sont des émulsions $\mathrm{L} / \mathrm{H}$. La formule de base ainsi que l'origine des matières premières sont présentées dans le tableau 1. Les émulsions sont réalisées à chaud à l'aide d'un bain-marie de type Memmert $\left(\mathrm{t}^{\circ}=75^{\circ} \mathrm{C}\right)$. Le mélange est réalisé en mode inverse, c'est-à-dire en incorporant la phase aqueuse à la phase grasse et ce sous agitation à I'aide d'un mélangeur Yellow line OST basic (15 minutes à 800 tours/minute ; 30 minutes à 500 tours/minute jusqu'à refroidissement). Cette méthode de préparation présente l'avantage d'augmenter la stabilité des préparations et limite les pertes.

Les émulsions sont conditionnées dans des flacons à prélèvement à cape à visser (Grosseron, St Herblain, France). Elles subissent une période de mûrissement de 24 heures. À partir de la formule de base, 18 émulsions ont été réalisées en incorporant un seul corps gras en remplacement des ingrédients de la phase grasse ( $20 \%$ de la formule). Le tableau 2 donne les caractéristiques des corps gras employés.

La stabilité de toutes les formules a été étudiée, d'une part à $40^{\circ} \mathrm{C}$ pendant 3 mois, d'autre part à $50^{\circ} \mathrm{C}$ pendant 1 mois (étuves Memmert, type UE-200) en déterminant les caractères organoleptiques, le $\mathrm{pH}$ à l'aide d'un
pH-mètre Basic 20 (Bioblock Scientific, Illkirch, France) équipé d'une électrode $\mathrm{pH}$ à rodage Inlab 420 et la viscosité grâce à un viscosimètre à mobile tournant (viscosimètre RVDV2 plus système Hélipath avec mobiles, Labomat, Saint Denis, France). Le contrôle de la propreté microbiologique des préparations a été mené selon la méthode recommandée par la Pharmacopée européenne, $5^{\mathrm{e}}$ édition (Ch. 2.6.12 2.6.13).

Le spectre ultraviolet de chacune des huiles diluée à $1 \%$ dans l'huile de paraffine a été réalisé grâce à un spectrophotomètre double faisceau (Hitachi UV-visible, model U-2000).

Pour déterminer leur SPF, les émulsions sont étalées à l'aide d'un doigtier par un même manipulateur sur des plaques de polyméthylméthacrylate (PMMA) de $25 \mathrm{~cm}^{2}$, préalablement nettoyées à l'éthanol. Le choix de ce type de matériau se justifie par le fait que l'on veut reproduire la surface cutanée. Le dépôt d'émulsion se fait à raison de $1,5 \mathrm{mg} / \mathrm{cm}^{2}$. L'étalement doit être le plus homogène possible. Les plaques sont laissées 15 minutes à l'obscurité. Puis, grâce un spectrophotomètre à sphère d'intégration (UV1000S, Laser 2000, SaintNom la Bretèche, France), nous déterminons le SPF du produit étudié.

Le calcul du SPF repose sur la méthode de Diffey et Robson [4] :

$$
S P F=\int_{290}^{320} E \lambda \cdot S \lambda d \lambda / \int_{290}^{320} E \lambda \cdot S \lambda \cdot T \lambda d \lambda
$$


Tableau 1. Composition centésimale de la crème de base.

\begin{tabular}{|c|c|c|c|}
\hline Nom commercial & Nom INCI & Fournisseur ville, pays & $\%$ \\
\hline $\begin{array}{l}\text { Abil WE } 09 \text { (Cosmetic } \\
\text { Grade) }\end{array}$ & $\begin{array}{l}\text { Polyglyceryl-4-isostearate ; } \\
\text { Cetyl dimethicone } \\
\text { copolyol ; hexyllaurate }\end{array}$ & $\begin{array}{l}\text { Goldschmidt, Essen, } \\
\text { Allemagne }\end{array}$ & 5,00 \\
\hline Huile de paraffine & Paraffinum liquidum & Cooper, Melun, France & 10,00 \\
\hline Cétiol HE & PEG-7 glyceryl cocoate & Cognis, Ponthierry, France & 5,00 \\
\hline Butylhydroxytoluène & BHT & $\begin{array}{l}\text { Lambert Rivière } \\
\text { Fontenay-sous-bois, France }\end{array}$ & 0,01 \\
\hline Acide stéarique & Stearic acid & Cooper, Melun, France & 5,00 \\
\hline Eumulgin B1 & Ceteareth-12 & $\begin{array}{l}\text { Sidobre Sinnova } \\
\text { St Fargeau, France }\end{array}$ & 1,50 \\
\hline Eumulgin B2 & Ceteareth-20 & $\begin{array}{l}\text { Sidobre Sinnova } \\
\text { St Fargeau, France }\end{array}$ & 1,50 \\
\hline Glycérine & Glycerin & Cooper, Melun, France & 4,00 \\
\hline Méthyl paraben sodé & Sodium methylparaben & $\begin{array}{l}\text { Merck, Darmstadt, } \\
\text { Allemagne }\end{array}$ & 0,10 \\
\hline Propyl paraben sodé & Sodium propylparaben & $\begin{array}{l}\text { Merck, Darmstadt, } \\
\text { Allemagne }\end{array}$ & 0,05 \\
\hline Blanose & Cellulose gum & Aqualon, Alizay, France & 0,90 \\
\hline Uvinul MC 80 & Octyl Methoxycinnamate & $\begin{array}{l}\text { BASF, Ludwigshafen, } \\
\text { Allemagne }\end{array}$ & 4,00 \\
\hline Triéthanolamine & Triethanolamine & Cooper, Melun, France & 0,30 \\
\hline Eau distillée & Aqua & $\begin{array}{l}\text { Production au LPiC - } \\
\text { Autostill }\end{array}$ & qsp 100 \\
\hline
\end{tabular}

Tableau 2. Les différents corps gras utilisés.

\begin{tabular}{|lll|}
\hline Corps gras & \multicolumn{1}{c|}{ Nom INCI } & \multicolumn{1}{c|}{ Fournisseur } \\
Nom commercial & & Cooper, Melun, France \\
\hline $\begin{array}{l}\text { Huile de noyaux d'abricots vierge } \\
\text { Huile de ricin vierge }\end{array}$ & $\begin{array}{l}\text { Prunus armeniaca } \\
\text { Huile de coco }\end{array}$ & Cooper, Melun, France \\
Huile de germes de maïs & Cocos nucifera & Cooper, Melun, France \\
Huile d'argan vierge & Argania spinosa seed oil & Alban Müller, Vincennes, France \\
Beurre de karité & Butyrospermum parkii & Alban Müller, Vincennes, France \\
Huile de rosier muscat raffinée & Rosa moschata & Sederma, Le Perray, France \\
Huile d'amande douce raffinée & Prunus dulcis & Alban Müller, Vincennes, France \\
Huile de jojoba vierge & Buxus chinensis & Cooper, Melun, France \\
Huile d'arachide raffinée & Arachis hypogaea & Soliance, Colombes, France \\
Blanc de baleine synthétique & Cetyl alcohol & Cooper, Melun, France \\
Huile de sésame vierge & Sesamum indicum & Cooper, Melun, France \\
Huile d'olive raffinée & & Michel Bertin, Lagny le Sec, \\
Palmotène & Olea europaea & France \\
Huile de pépins de cassis raffinée & Ribes nigrum & Michel Bertin, Lagny le Sec, \\
Huile de carthame désodorisée et & Carthamus tinctorius & France \\
raffinée & Lanolin & Michel Bertin, Lagny le Sec, \\
Lanoline & & France \\
Abil WE 09 & Alban Müller, Vincennes, France \\
\hline
\end{tabular}

avec $\mathrm{E}_{\lambda}$ efficacité érythémateuse spectrale (CIE), $S_{\lambda}$ l'irradiance spectrale solaire et $T_{\lambda}$ la transmittance spectrale de l'échantillon.

Neuf points de mesure sont effectués par plaque et trois plaques sont requises par produit. Les 27 valeurs obtenues sont alors traitées statistiquement.

\section{Résultats et discussion}

Le $\mathrm{pH}$ des différentes préparations est égal à 7 . La viscosité est de $135000 \mathrm{mPa} . \mathrm{s}^{\mathrm{a}} 20^{\circ} \mathrm{C}$. Aucune modification significative n'ayant été observée dans le temps et dans les conditions de conservation considérées, nous concluons à une bonne stabilité des formules réalisées.

L'étude des spectres ultraviolets nous fournit la valeur de la longueur d'onde maximale $\mathrm{d}^{\prime}$ absorption $\left(\lambda_{\max }\right)$. Cependant la simple utilisation d'un spectrophotomètre ne peut prétendre à l'obtention du SPF. En effet, chaque ingrédient se retrouve après l'étape de formulation au sein d'un mélange complexe où peuvent se produire de multiples interactions. Il est d'ailleurs bien connu que l'association de certains filtres chimiques est déconseillée, sachant que leur effet n'est pas additif. Ce que l'on désire connaître, c'est l'effet photoprotecteur $d^{\prime} u n e$ formule et non le spectre d'une matière première isolée, qui peut nous renseigner toutefois sur ses potentialités.

La méthode de détermination des SPF in vitro à I'aide des plaques de PMMA semble tout à fait satisfaisante. En effet, ces plaques sont compatibles avec tout type d'excipient et elles reproduisent au mieux les propriétés physiologiques et optiques de la peau [5].

L'émulsion de base renfermant $4 \%$ d'octylméthoxycinnamate présente un SPF de $8,63 \pm 0,65$. L'influence des corps gras étudiés sur ce SPF varie en fonction de la nature du corps gras (tableau 3). L'intervalle de confiance a été calculé à l'aide du test $t$ de Student $(P=0,95, n=27)$. Ce dernier permet de classer les corps gras étudiés en trois catégories (figure 1). La lanoline apparaît particulièrement intéressante, même si elle est réputée allergisante [6]. L'huile de sésame s'avère un bon exhausteur de SPF et peut constituer un bon ingrédient pour les propriétés antisolaires, d'autant que des propriétés protectrices vis-àvis du stress oxydatif viennent d'être mises en évidence chez l'animal [7]. Dans la même catégorie, nous citerons des huiles aux propriétés émollientes: huiles de pépins de cassis, $d^{\prime}$ argan et d'amande douce. Le beurre de karité, du fait de sa composition ( $\alpha$ et $\beta$ amyrine, deux esters cinnamiques), laissait espérer des résultats meilleurs $[8,9]$. Or il ne s'avère pas plus intéressant que I'huile de ricin, par exemple. L'huile de silicone, si elle améliore l'étalement ou la substantivité, a peu d'effet sur le SPF 
Tableau 3. Influence du corps gras sur le SPF de la préparation antisolaire.

\begin{tabular}{|lccc|}
\hline Corps gras étudié & SPF moyen & Ecart-type & $\begin{array}{c}\text { Intervalle } \\
\text { de confiance }\end{array}$ \\
\hline Lanoline & 16,88 & 1,30 & $16,36-17,39$ \\
Huile de pépins de cassis & 14,97 & 0,78 & $14,66-15,28$ \\
Huile d'argan & 14,92 & 0,81 & $14,60-15,24$ \\
Huile de germes maïs & 14,87 & 2,18 & $14,00-15,73$ \\
Huile d'amande douce & 14,79 & 0,75 & $14,49-15,09$ \\
Huile de sésame & 14,78 & 1,49 & $14,19-15,37$ \\
Huile d'arachide & 14,78 & 0,70 & $14,50-15,06$ \\
Palmotène & 14,56 & 0,99 & $14,17-14,95$ \\
Huile de carthame & 14,27 & 0,31 & $14,15-14,39$ \\
Huile de jojoba & 13,67 & 0,98 & $13,28-14,06$ \\
Huile de noyaux d'abricots & 13,40 & 1,63 & $12,76-14,04$ \\
Beurre de karité & 13,30 & 1,39 & $12,75-13,85$ \\
Huile d'olive & 13,29 & 1,50 & $12,70-13,88$ \\
Huile de ricin & 12,77 & 2,35 & $11,84-13,70$ \\
Huile de silicone & 12,31 & 0,94 & $11,94-12,68$ \\
Blanc de baleine & 12,22 & 2,04 & $11,41-13,03$ \\
Huile de coco & 11,68 & 2,03 & $10,88-12,48$ \\
Huile de rosier muscat & 11,61 & 2,13 & $10,77-12,45$ \\
\hline
\end{tabular}

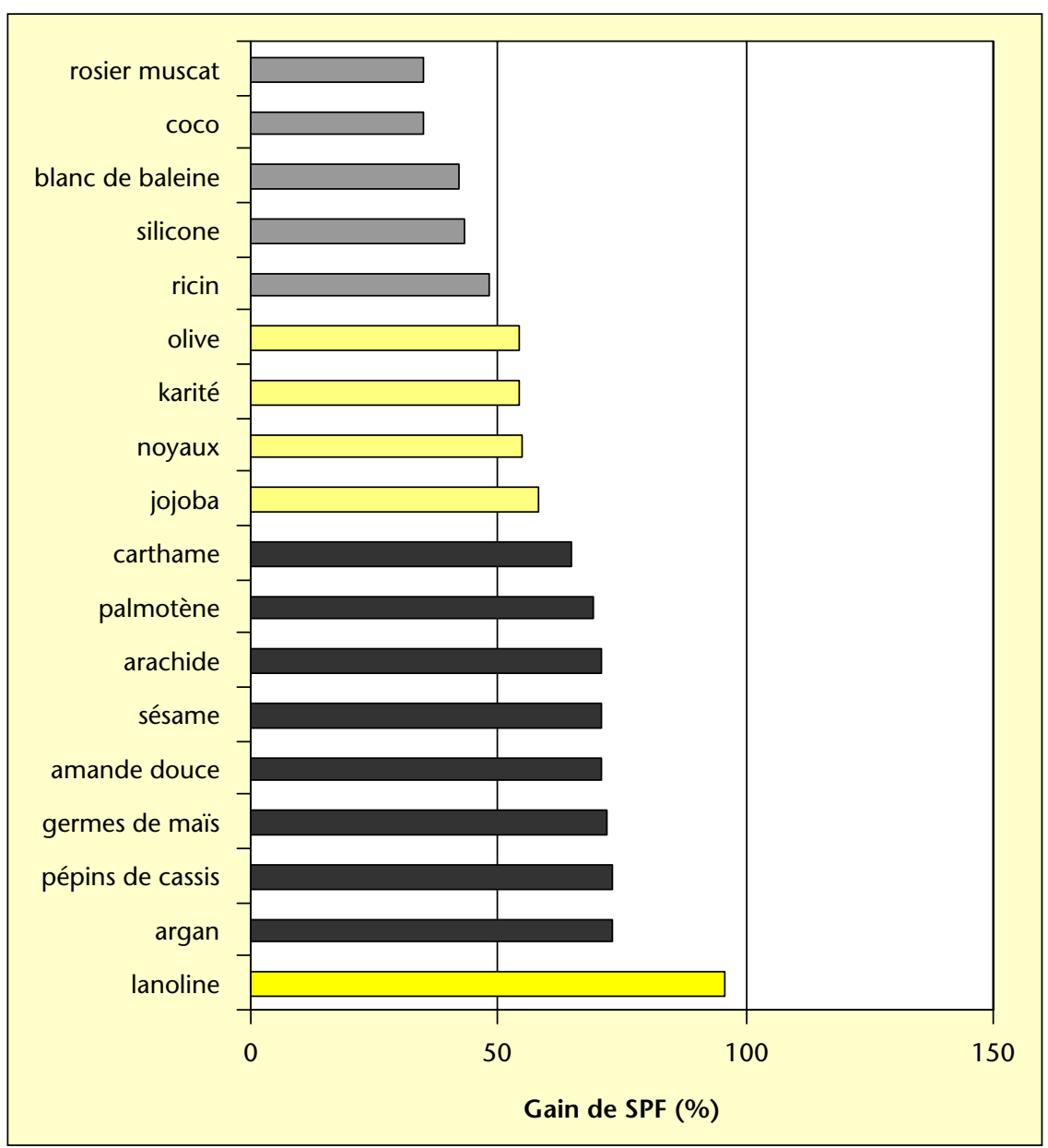

Figure 1. Intérêt de différents corps gras dans la formulation de produits antisolaires. lui-même, ce que laissait déjà supposer son spectre UV.

Il serait intéressant de connaître l'influence du pourcentage de corps gras, en incorporant des quantités supérieures. Cependant de nouveaux essais de stabilité seraient nécessaires. II faudrait aussi évaluer les modifications de la texture et l'augmentation du coût de la formule.

\section{RÉFÉRENCES}

1. SCHULZE R. Einige Versuche und Bemerkungen zum Problem der hendelsüblichen Lichtschutzmittel. Parfüm kosmet 1956 ; 37 : 310-5.

2. STOCKES R, DIFFEY B. How well are sunscreen user protected? Photodermatol Photoimmunol Photomed $1997 ; 13$ : 186-8.

3. SUTTON JD. The sunscreening effect of topical sunflower oil. J Am Acad Dermatol $1983 ; 8(6)$ : 909-10.

4. DIFFEY BL, ROBSON J. A new substrate to measure sunscreen protection factor throughout the ultraviolet spectrum. / Soc Cosmet Chem $1989 ; 40$ : 127-33.

5. LUTZ D. Contribution to measuring in vitro protection. In : Proceedings of the 2nd European UV sunfilters conference 1999 : 26-39.

6. KLIGMAN AM. Lanolin allergy: crisis or comedy. Contact Dermatitis 1983; 9(2): 99-107.

7. HSU D, LIU MY. Sesame oil protects against lipopolysaccharide- stimulated oxidative stress in rats. Crit Care Med 2004 ; 32(1) : 227-31.

8. ITOH T, MATSUMOTO T, TAMURA A. Sterols and methylsterols and triterpene alcohol in some vegetable oils. Lipids $1974 ; 9$ : 173-83.

9. DI VINCENZO D, MARANZ S, SERRAIOCCO A, VITO R, WIESMAN Z, BIANCHI G. Regional variation in shea butter lipid and triterpene composition in four African countries. / Agric Food Chem 2005 ; 53 : 7473-9. 